\title{
Recent heavy-flavour results from ATLAS
}

\author{
Jing Chen On behalf of the ATLAS Collaboration ${ }^{1, \star}$ \\ ${ }^{1}$ University of Science and Technology of China, Department of Modern Physics, 96 Jinzhai Road, Anhui \\ Hefei, China
}

\begin{abstract}
The ATLAS heavy-ion program utilizes heavy-flavour hadrons to probe the hot, dense matter formed at the LHC. Quarkonium measurements have been performed in $p p, p+\mathrm{Pb}$ and $\mathrm{Pb}+\mathrm{Pb}$ systems to study medium effects. The $\mathrm{Pb}+\mathrm{Pb}$ results show a strong suppression of charmonium productions in more central events. Proton-lead interactions show little modification of the $1 \mathrm{~S}$ charmonium state, but seem to indicate a centrality dependence of the $2 \mathrm{~S}$ state. Upsilons have been studied in $p+\mathrm{Pb}$ and are found to show decreasing behaviour in more central collisions. Inclusive muons with $p_{\mathrm{T}}$ above $4 \mathrm{GeV}$ have been studied to provide insight on open-flavour production, and are found to be strongly suppressed in $\mathrm{Pb}+\mathrm{Pb}$ collisions with a substantial and significant elliptic flow signal. Muon-hadron correlations have also been studied in the $2016 \sqrt{s_{N N}}=8.16$ $\mathrm{TeV} p+\mathrm{Pb}$ data. There are clear indications of a near-side ridge, suggesting that similar mechanisms may be relevant for both the small and large systems.
\end{abstract}

\section{Introduction}

Measurements of quarkonium production and the azimuthal anisotropy of hadron production in $p+\mathrm{Pb}$ and $\mathrm{Pb}+\mathrm{Pb}$ collisions provide opportunites to understand the cold nuclear matter (CNM) effects and the properties of hot and dense medium, quark gluon plasma (QGP), created in heavy-ion collisions. This paper presents a few measurements of this kind carried out using datasets collected by the ATLAS detector [1] from 2013 to 2016 in $p p, p+\mathrm{Pb}$ and $\mathrm{Pb}+\mathrm{Pb}$ collisions at the LHC. In this paper, the following topics are discussed: 1) the measurement of nuclear modification factor of quarkonium production in $p+\mathrm{Pb}$ and $\mathrm{Pb}+\mathrm{Pb}$ collisions $[2,3]$; 2) the measurement of azimuthal anisotropy of charged particles and muons [4]. Among CNM effects, modification of nPDFs, nuclear absorption, induced medium energy loss and gluon saturation can be quantified by the nuclear modification factor, the ratio of quarkonium production cross sections in $\mathrm{A}+\mathrm{A}$ relative to the cross sections measured in $p p$ collisions at the same centre-of-mass energy. For excited quarkonium states, double ratio (the ratio of the nuclear modification factor in excited quarkonium states to the nuclear modification factor in the ground state) often to be used to quantify the CNM effects. The azimuthal anisotropy in particle $d N / d \phi$ distributions, usually described by a Fourier coefficient with $n^{\text {th }}$ order harmonic $\left\langle\cos \left[n\left(\phi-\Psi_{n}\right)\right]\right\rangle, v_{n}$, where $\phi$ is the azimuthal angle of the partical momentum, and $\Psi_{n}$ is the experimental event plane angle of $n^{\text {th }}$ order. These measurements can provide valuable constraints on QGP transport property.

\footnotetext{
^e-mail: jing.chen@cern.ch
} 


\section{Nuclear modification factor}

It is well-known that $J / \psi$ and $\psi(2 S)$ can be produced from short-lived QCD decays including feeddown from other charmonium states (prompt) and produced in the decays of long lived $b$-hadrons (non-prompt). In this paper the measurements of prompt and non-prompt $J / \psi, \psi(2 \mathrm{~S})$ and $\Upsilon$ production are presented. Measurement of $J / \psi, \psi(2 \mathrm{~S})$ and $\Upsilon(n \mathrm{~S})$ production using $28 \mathrm{nb}^{-1}$ of $p+\mathrm{Pb}$ data at $\sqrt{s_{N N}}=5.02 \mathrm{TeV}$ and $25 \mathrm{pb}^{-1}$ of $p p$ data at centre-of-mass energy of $\sqrt{s}=5.02 \mathrm{TeV}$ [2]. Measurement of $J / \psi$ and $\psi(2 \mathrm{~S})$ productions in $0.42 \mathrm{nb}^{-1}$ of $\mathrm{Pb}+\mathrm{Pb}$ data at $\sqrt{S_{N N}}=5.02 \mathrm{TeV}$ and $25 \mathrm{pb}^{-1}$ of $p p$ data at centre-of-mass energy of $\sqrt{s}=5.02 \mathrm{TeV}$ are also presented [3]. The nuclear modification factor:

$$
\begin{aligned}
R_{\mathrm{AA}} & =\frac{N_{\mathrm{AA}}}{\left\langle T_{\mathrm{AA}}\right\rangle \times \sigma^{p p}} \\
R_{p \mathrm{~Pb}} & =\frac{N_{p \mathrm{~Pb}}}{\left\langle T_{p \mathrm{~Pb}}\right\rangle \times \sigma^{p p}}
\end{aligned}
$$

where $N_{\mathrm{AA}}\left(N_{p \mathrm{~Pb}}\right)$ is the per event yield of quarkonium states measured in $\mathrm{A}+\mathrm{A}(p+\mathrm{Pb})$ collisions, $\left\langle T_{\mathrm{AA}}\right\rangle\left(\left\langle T_{p \mathrm{~Pb}}\right\rangle\right)$ is the mean nuclear thickness function and $\sigma^{p p}$ is the cross section for the corresponding quarkonium states in $p p$ collisions at the same energy. Figure 1 and Figure 2 show the modification factor for $J / \psi$. In $p+\mathrm{Pb}$ collisions, no obvious $p_{\mathrm{T}}$ and $y^{*}$ (centre-of-mass rapidity) dependence is observed. In $\mathrm{Pb}+\mathrm{Pb}$ results, for prompt $J / \psi$, there is a trend of slight increase in $R_{\mathrm{AA}}$ with increasing $p_{\mathrm{T}}$. For non-prompt $J / \psi, R_{\mathrm{AA}}$ is almost a constant as a function of $p_{\mathrm{T}}$ within the uncertainties. Both prompt and non-prompt $J / \psi R_{\mathrm{AA}}$ are essentially flat as a function of rapidity. $J / \psi$ production is most strongly suppressed in central collisions.
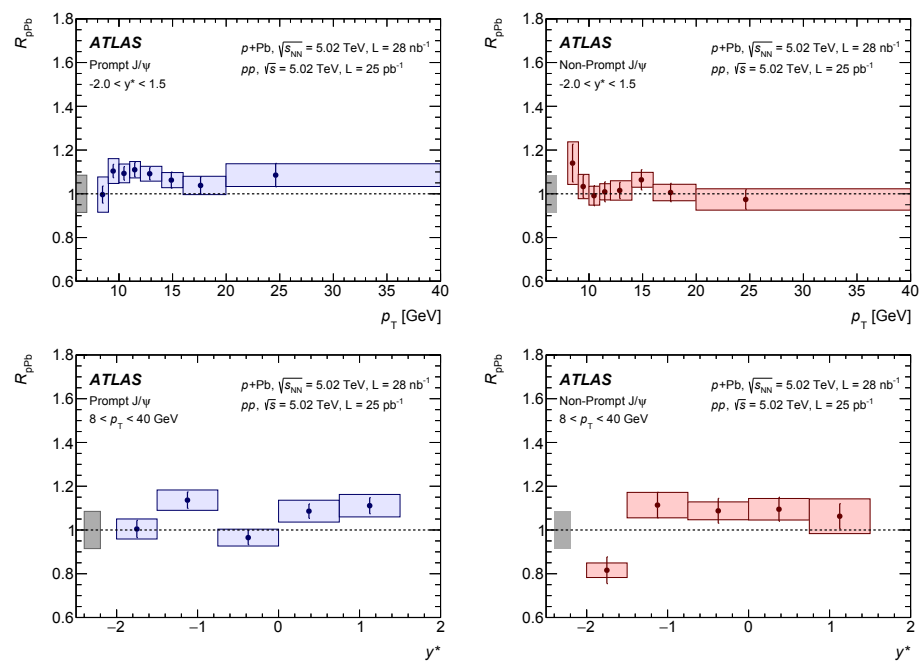

Figure 1. The nuclear modification factor, $R_{p \mathrm{~Pb}}$, as a function of $p_{\mathrm{T}}$ (top) and $y^{*}$ (bottom) for the production of prompt $J / \psi$, non-prompt $J / \psi$. The vertical error bars correspond to the statistical uncertainties [2].

In Figure 3, the $\Upsilon(n S)(n=2,3)$ to $\Upsilon(1 \mathrm{~S})$ double ratio provides information on the difference of the CNM effects on states with different binding energies. Both the integrated double ratio are found to be less than unity by two standard deviations. For the double ratio as a function of centrality, both 

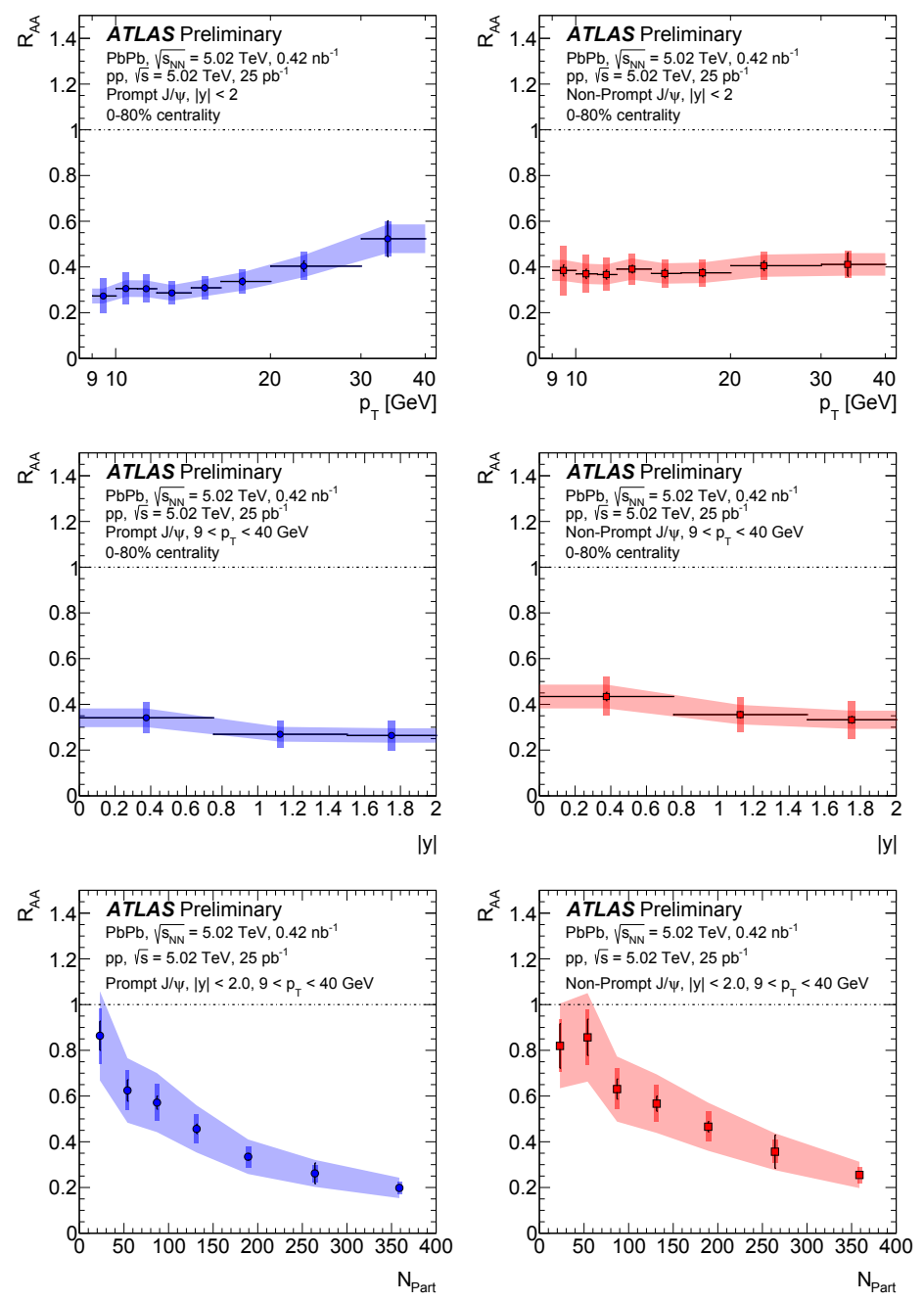

Figure 2. The nuclear modification factor as a function of $p_{\mathrm{T}}$ (top), rapidity y (middle) and centrality (bottom) for the prompt $J / \psi$ (left) and non-prompt $J / \psi$ (right). The statistical uncertainty on each point is indicated by a narrow error bar which is smaller than the plotting symbol for most points. The wide error bar plotted with each point represents the point-to-point systematic uncertainty, while the error band connecting the points represents correlated scale uncertainties [3].

are found to decrease slightly with increasing centrality at the significance level of one-standarddeviation. The suppression of double ratio for prompt $\psi(2 \mathrm{~S})$ and $J / \psi$ indicates the more tightly bound quarkonium system, the $J / \psi$, survives the temperature of the hot dense medium with a higher probability than the more loosely bound quarkonium, the $\psi(2 S)$. Non-prompt mesons are always formed outside the medium, thus, the double ratio is expected to be unity, as is observed. 

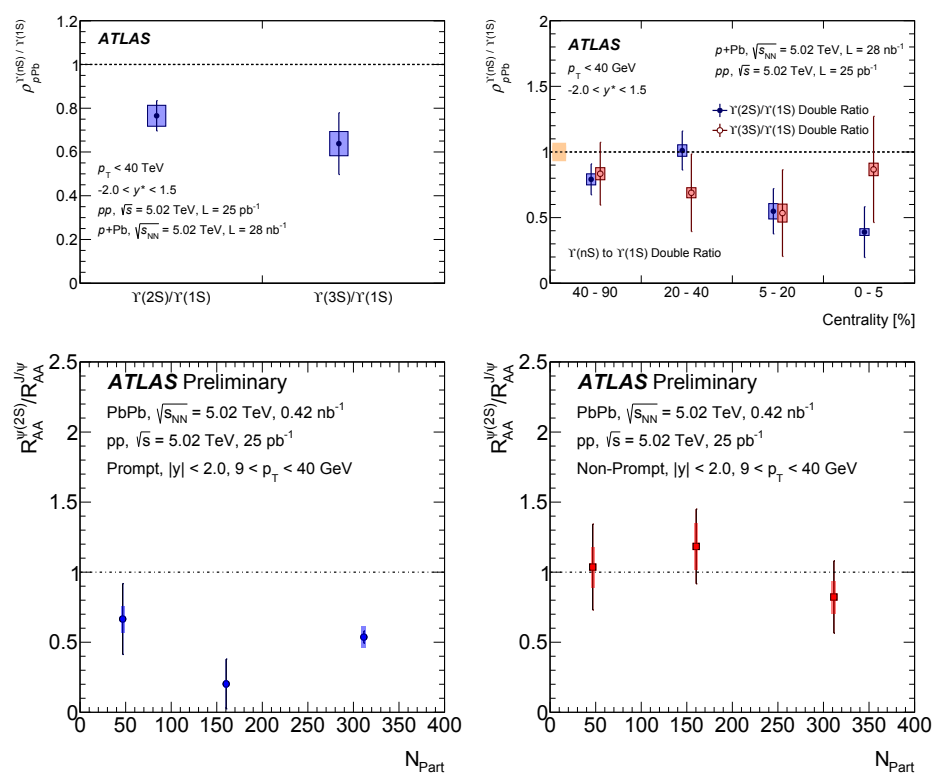

Figure 3. Top: The $\Upsilon(n \mathrm{~S})(n=2,3)$ to $\Upsilon(1 \mathrm{~S})$ double ratio, integrated in the whole measured $p_{\mathrm{T}}$ and $\mathrm{y}^{*}$ range (left). The $\Upsilon(n \mathrm{~S})(n=2,3)$ to $\Upsilon(1 \mathrm{~S})$ double ratio, as a function of event centrality in $p+\mathrm{Pb}$ collisions [2]. Bottom: double ratio for $\psi(2 \mathrm{~S})$ to $J / \psi$, as a function of centrality expressed as $N_{\text {Part }}$, for prompt meson production (left) and non-prompt meson production (right). This ratio has the advantage that corrections are similar for the numerator and denominator, and thus systematic uncertainties are reduced. The narrow error bar represents the statistical uncertainties while the wider error bar represents the total systematic uncertainty [3].

\section{Azimuthal anisotropy}

For a given event class, the two particle correlation function is defined as [4]:

$$
C(\Delta \eta, \Delta \phi)=\frac{S(\Delta \eta, \Delta \phi)}{B(\Delta \eta, \Delta \phi)}
$$

where $\Delta \phi \equiv \phi^{a}-\phi^{b}$ is the relative azimuthal angle, $\Delta \eta \equiv \eta^{a}-\eta^{b}$ is the relative pseudorapidity. $S$ and $B$ represent pair distributions constructed from the same event and from mixed event. One-dimensional correlation function $C(\Delta \phi)$ is obtained by integrating Eq. (3). Figure 4 shows the two-particle h$\mu$ correlation functions in $8.16 \mathrm{TeV} p+\mathrm{Pb}$ collisions. A significant contribution to the two-particle corelations arise from back to back dijets is observed. In order to estimate the contribution of such dijets and other process, a template fit $C^{\text {templ }}(\Delta \phi)$ is used:

$$
\begin{aligned}
& C^{\text {templ }}(\Delta \phi)=F C^{\text {periph }}(\Delta \phi)+C^{\text {ridge }}(\Delta \phi) \\
& C^{\text {ridge }}(\Delta \phi)=G\left(1+\sum_{n=2}^{\infty} 2 v_{n, n} \cos (n \Delta \phi)\right)
\end{aligned}
$$

where $F$ is the scale factor, $C^{\text {periph }}(\Delta \phi)$ is the correlation measured in low multiplicity or "peripheral" events, $C^{\text {ridge }}(\Delta \phi)$ is the genuine long-range harmonic modulation. In Eq. (5), $G$ represents the mag- 
nitude of the combinatoric component of $C^{\text {ridge }}(\Delta \phi)$. The harmonics $v_{n, n}$ quantify the strengh of the genuine long-range correlation. The single-particle anisotropies $v_{n}$ can be obtained from $v_{n, n}$ :

$$
v_{n, n}\left(p_{\mathrm{T}}^{a}, p_{\mathrm{T}}^{b}\right)=v_{n}\left(p_{\mathrm{T}}^{a}\right) v_{n}\left(p_{\mathrm{T}}^{b}\right)
$$

where $\mathrm{a}$ and $\mathrm{b}$ are two particles in the long-range correlations.
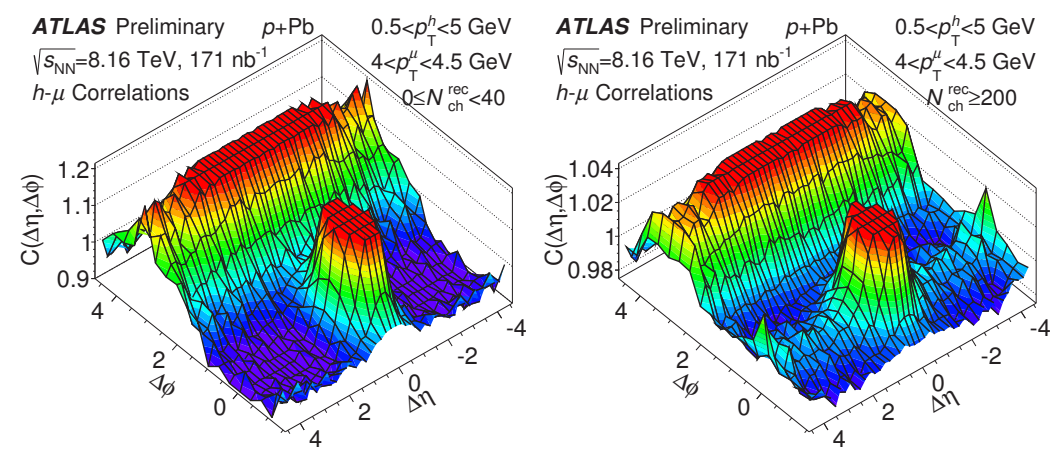

Figure 4. Two-particle $\mathrm{h}-\mu$ correlation functions in $\sqrt{s_{N N}}=8.16 \mathrm{TeV} p+\mathrm{Pb}$ collisions [4].
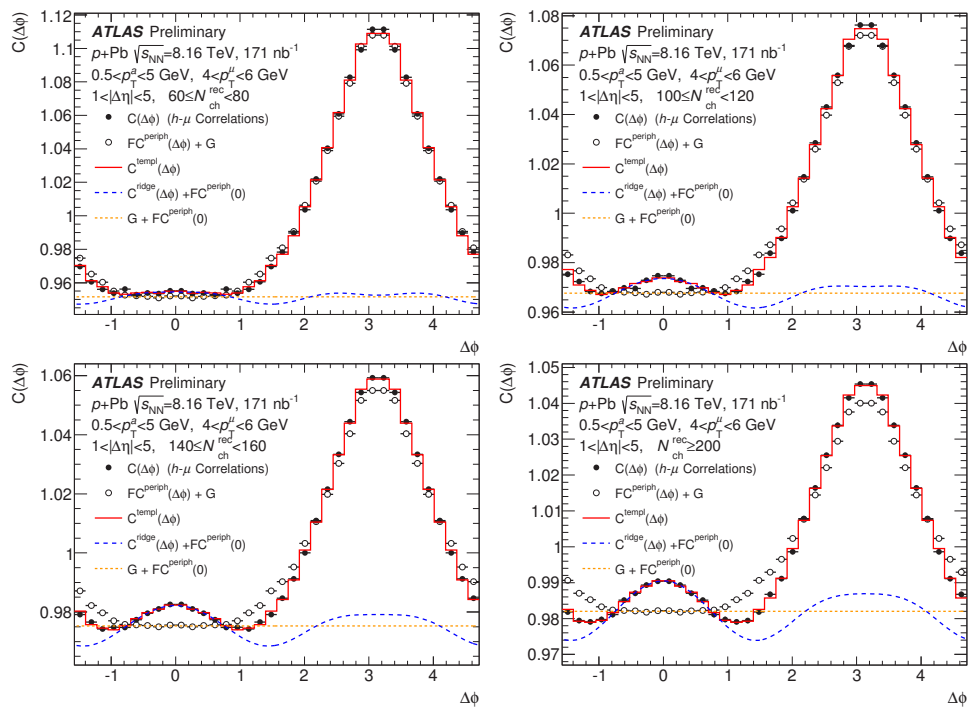

Figure 5. Template fits to h- $\mu$ correlations for $|\Delta \eta|>1$. Each panel corresponds to a different multiplicity interval [4].

Figure 5 shows template fits to $h-\mu$ correlations for $|\Delta \eta|>1$. Each panel corresponds to a different multiplicity interval. The template fitting includes second-order, third-order and fourth-order harmonics. Measurements of heavy flavour muons in $\mathrm{Pb}+\mathrm{Pb}$ and $p p$ collisions show an elliptic anisotropy [5]. 
Such correlations were understood to be the result of azimuthal anisotropies of the single-particle distributions. In $p+\mathrm{Pb}$ analyses of long-range correlations, the event activity is quantified by $N_{\mathrm{ch}}^{\mathrm{rec}}:$ the total number of reconstructed charged-particle tracks passing the track selections [4]. In Figure 6, the measured $v_{2}$ for charged particles increases with $N_{\mathrm{ch}}^{\mathrm{rec}}$ at low $N_{\mathrm{ch}}^{\mathrm{rec}}$ and eventually saturates for $N_{\mathrm{ch}}^{\mathrm{rec}}$ $>150$. However even at the lowest measured multiplicity, a large $v_{2}$ is observed. Over the $p_{\mathrm{T}}$ range shown, the measured $v_{2}$ for muons are consistent with having no $N_{\mathrm{ch}}^{\mathrm{rec}}$ dependence.
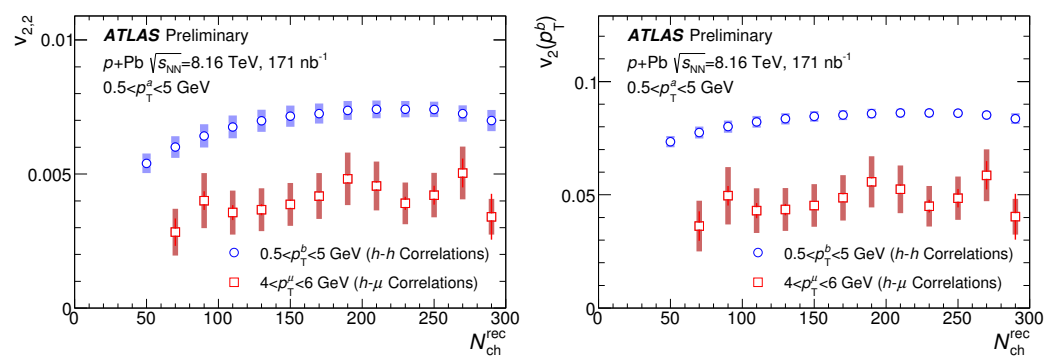

Figure 6. The $v_{2,2}$ to h-h correlations (circles), h- $\mu$ correlations (squares) and the corresponding $v_{2}$ values for the associated particle [4].

\section{Conclusion}

ATLAS has measured the production of quarkonia and heavy flavour muons in $p+\mathrm{Pb}$ and $\mathrm{Pb}+\mathrm{Pb}$ systems. For quarkonium production, stronger suppression in more central $\mathrm{Pb}+\mathrm{Pb}$ collisions is observed. Proton-lead interactions show little modification of the ground charmonium state. Upsilons also have been studied in $p+\mathrm{Pb}$ and are found to show decreasing behaviour in more central collisions. Significant azimuthal flow modulation of heavy flavour muon is observed via heavy flavour muon-hadron correlation. These results can provide insight into nuclear parton distributions, allow quantitative studies of heavy quarkoium production, and also provide the opportunity to improve our understanding of the propagation of heavy quarks in the QGP.

\section{References}

[1] ATLAS Collaboration, JINST 3, S08003 (2008)

[2] ATLAS Collaboration (2017), arXiv: 1709.03089

[3] ATLAS Collaboration, ATLAS-CONF-2016-109 (2016), https://cds.cern.ch/record/ 2220771

[4] ATLAS Collaboration, ATLAS-CONF-2017-006 (2017), https://cds.cern.ch/record/ 2244808

[5] ATLAS Collaboration, ATLAS-CONF-2015-053 (2015), https://cds.cern.ch/record/ 2055674 